\title{
Vacuum Lightcone Fluctuations in a Dielectric
}

\author{
C. H. G. Bessa, ${ }^{1, *}$ V. A. De Lorenci, ${ }^{2,1, \dagger}$ L. H. Ford, ${ }^{1,}+\underset{\text { and N. F. Svaiter }}{3, \S}$ \\ ${ }^{1}$ Institute of Cosmology, Department of Physics and Astronomy \\ Tufts University, Medford, Massachusetts 02155, USA \\ ${ }^{2}$ Instituto de Física e Química, Universidade Federal de Itajubá \\ Itajubá, Minas Gerais 37500-903, Brazil \\ ${ }^{3}$ Centro Brasileiro de Pesquisas Físicas \\ Rua Dr. Xavier Sigaud 150, 22290-180 Rio de Janeiro, RJ, Brazil
}

\begin{abstract}
A model for observable effects of time modulated electromagnetic vacuum fluctuations is presented. The model involves a probe pulse which traverses a slab of nonlinear optical material with a nonzero second order polarizability. We argue that the pulse interacts with the ambient vacuum fluctuations of other modes of the quantized electric field, and these vacuum fluctuations cause variations in the flight time of the pulse through the material. The geometry of the slab of material defines a sampling function for the quantized electric field, which in turn determines that vacuum modes whose wavelengths are of the order of the thickness of the slab give the dominant contribution. Some numerical estimates are made, which indicate that fractional fluctuations in flight time of the order of $10^{-8}$ are possible in realistic situations. The model presented here is both an illustration of a physical effect of vacuum fluctuations occurring in a finite interval of time, and an analog model for the lightcone fluctuations predicted by quantum gravity.
\end{abstract}

Keywords: switched vacuum fluctuations, lightcone fluctuations, variable light propagation speed, nonlinear dielectrics. analog model for quantum gravity, vacuum electric field fluctuations

\footnotetext{
*Electronic address: carlos@cosmos.phy.tufts.edu

${ }^{\dagger}$ Electronic address: delorenci@unifei.edu.br

${ }^{\ddagger}$ Electronic address: ford@cosmos.phy.tufts.edu

$\S$ Electronic address: nfuxsvai@cbpf .br
} 


\section{INTRODUCTION}

Vacuum fluctuations of the electromagnetic field are responsible for several observed phenomena, including the Lamb shift and the Casimir effect. However, these effects are typically energy level shifts in stationary systems, not events which can be localized in time. The dynamical Casimir effect, which has been observed in superconducting circuits [1], does involve time dependence. However, it is not really a vacuum fluctuation effect in the sense of the static Casimir effect. Rather, the dynamical Casimir effect is quantum creation of particles by a time dependent background, and is essentially the same effect as particle creation in an expanding universe [2], or quantum radiation by moving mirrors [3-5].

There is still debate about the reality of vacuum fluctuations as temporal events [6, 7]. Here we explore the viewpoint that vacuum fluctuations can be just as real as thermal fluctuations, but are often not noticed because of strong anticorrelations. The anticorrelations prevent an electric charge from undergoing observable Brownian motion in the vacuum state. The charge can temporarily acquire energy from a vacuum electric field fluctuation, but this energy will be taken away by an anticorrelated fluctuation on a time scale consistent with the energy-time uncertainty principle. This viewpoint is supported by calculations in models where the cancellation is upset by a time dependent background [8-11]. We will here construct a model without an explicit external time dependent background, but where vacuum electric field fluctuations on a finite time scale have a clear, and potentially observable, physical effect.

Let $E$ be a Cartesian component of the quantized electric field operator. Vacuum expectation values of even powers of $E$ are divergent due to the contribution of high frequency modes. This divergence may be removed by replacing $E$ by its time average with a suitable sampling function, or test function, $f_{\tau}(t)$, where $\tau$ is the characteristic width of the function. Let

$$
\bar{E}=\int_{-\infty}^{\infty} E(t) f_{\tau}(t) d t
$$


Here $E(t)$ is the field operator at any fixed space point, and

$$
\int_{-\infty}^{\infty} f_{\tau}(t) d t=1
$$

The moments of $\bar{E}$ are finite and those of a Gaussian distribution, determined by the second moment

$$
\left\langle 0\left|\bar{E}^{2}\right| 0\right\rangle=\frac{a}{\tau^{4}}
$$

where the numerical constant $a$ depends upon the choice of sampling function. (LorentzHeaviside units with $c=\hbar=1$ will be used here, except as otherwise noted. In these units, electric field has dimensions of inverse time squared or inverse length squared.) For the case of a Lorentzian function,

$$
f_{\tau}(t)=\frac{\tau}{\pi\left(t^{2}+\tau^{2}\right)},
$$

we have $a=1 /\left(16 \pi^{2}\right)$. Modes whose period is of order $\tau$ give the dominant contribution here, with the contribution of shorter wavelength suppressed by the time averaging. This may be seen by noting that the time averaging replaces the sinusoidal time dependence, $\exp (-i \omega t)$, of a mode function with the Fourier transform of the sampling function, which is $\exp (-\omega \tau)$ in the case of the Lorentzian function. In rigorous treatments of quantum field theory, test functions, usually with compact support, are used to define well-behaved operators. See, for example, Ref. [12]. However, this use of test functions is purely formal, and no physical interpretation is made. One of the purposes of this paper will be to provide an example where the function $f_{\tau}(t)$ has a clear meaning defined by the physical system of interest. A different physical example was recently given in Ref. [13], which discussed the role of switched electric field fluctuations upon barrier penetration by an electron.

Our model will involve light propagation in a nonlinear material. Related models were presented in Ref. [14], as an analog model for semiclassical gravity, and in Ref. [15], as a model for the lightcone fluctuations expected in quantum gravity [16-21]. More details of the parallels between nonlinear optics and gravity were discussed in Refs. [14, 15]. Reference [15] dealt with fluctuations produced by photons in a squeezed state. In the present paper, the fluctuations will be vacuum fluctuations of the quantized electromagnetic field. 
In Sec. II we discuss some basic aspects of nonlinear optics and light propagation, and show how the flight time for a slab of material depends of the vacuum background field. Some aspects of the quantized electric field are treated in Sec. III. We show, by a simple argument in Sec. III A, how the correlation function in a nondispersive material depends upon the refractive index of the material. The relative magnitudes of $\left\langle 0\left|\bar{E}^{2}\right| 0\right\rangle$ and the mean squared field in a probe pulse are treated in Sec. III B. There it is argued that the mean squared field due to vacuum fluctuations can be larger than that of the classical probe pulse. In Sec. IV we calculate the flight time variance and give some explicit numerical estimates of the magnitude of the effect. Our results are summarized and discussed in Sec. V.

\section{NONLINEAR OPTICS AND LIGHT PROPAGATION}

In this section we discuss some basic aspects of light propagation in a nonlinear material, and give the key assumptions of our model. The polarization is a nonlinear function of the electric field which can be written as [22]

$$
P_{i}=\left(\chi_{i j}^{(1)} E_{j}+\chi_{i j k}^{(2)} E_{j} E_{k}+\cdots\right)
$$

where repeated indices are summed upon. Here $\chi_{i j}^{(1)}$ and $\chi_{i j k}^{(2)}$ are the first and second order susceptibility tensors, respectively. The second and higher order susceptibilities lead to a nonlinear wave equation for the electric field. In this paper, we consider only first and second order susceptibilities. The nonlinear wave equation for the electric field may be expressed as

$$
\nabla^{2} E_{i}-\frac{\partial^{2} E_{i}}{\partial t^{2}}=\frac{\partial^{2}}{\partial t^{2}}\left(\chi_{i j}^{(1)} E_{j}+\chi_{i j k}^{(2)} E_{j} E_{k}\right) .
$$

See Ref. [15] for more details. We assume that the total electric field may be written as the sum of a background field $\mathbf{E}_{0}$ and a smaller but more rapidly varying probe field $\mathbf{E}_{1}$,

$$
\mathbf{E}=\mathbf{E}_{0}+\mathbf{E}_{1}
$$

where $\left|E_{1}\right| \ll\left|E_{0}\right|$ but $\left|\dot{E}_{1} / E_{1}\right| \gg\left|\dot{E}_{0} / E_{0}\right|$. Both $\mathbf{E}_{0}$ and $\mathbf{E}_{1}$ satisfy nonlinear equations, with a coupling term between them. However, if the second order susceptibility is sufficiently 
small, we may take the equation for $\mathbf{E}_{0}$ to be approximately linear. In the present paper, the background field describes the vacuum modes of the quantized electric field, and will be approximated as a linear field in an approximately isotropic material, so we set

$$
\chi_{i j}^{(1)} \approx \delta_{i j} \chi_{b}^{(1)}
$$

in the equation for $\mathbf{E}_{0}$. We are assuming that the modes which will give the dominant contribution to the relevant vacuum fluctuations are in a frequency range where dispersion may be neglected, and where the linear susceptibility is $\chi_{b}^{(1)}$. With these assumptions, the vacuum modes approximately satisfy the linear equation

$$
\nabla^{2} \mathbf{E}_{0}-\frac{1}{v_{b}^{2}} \frac{\partial^{2} \mathbf{E}_{0}}{\partial t^{2}}=0
$$

where

$$
v_{b}=\frac{1}{\sqrt{1+\chi_{b}^{(1)}}}=\frac{1}{n_{b}},
$$

where $v_{b}$ is the phase velocity of the vacuum modes, which experience an index of refraction of $n_{b}$.

We may also derive the equation satisfied by the probe field $\mathbf{E}_{1}$ from the equation for the full electric field, Eq. (6). If we write out this equation first with $\mathbf{E}=\mathbf{E}_{0}+\mathbf{E}_{1}$, then with $\mathbf{E}=\mathbf{E}_{0}$, and take the difference, the equation for $\mathbf{E}_{1}$ is the result. Next we may drop terms in this equation which are quadratic in $\mathbf{E}_{1}$, and which involve time derivatives of $\mathbf{E}_{0}$. The latter step depends upon our assumption that the background field varies more slowly than does the probe field. The result is the linear equation

$$
\nabla^{2} E_{1}^{i}-\frac{\partial^{2} E_{1}^{i}}{\partial t^{2}}=\chi_{i j}^{(1)} E_{1}^{j}+\left(\chi_{i j k}^{(2)}+\chi_{i k j}^{(2)}\right) E_{0}^{j} \frac{\partial^{2} E_{1}^{k}}{\partial t^{2}}
$$

We again assume that the first order susceptibility is isotropic, and set

$$
\chi_{i j}^{(1)} \approx \delta_{i j} \chi_{p}^{(1)}
$$

in Eq. (11), where $\chi_{p}^{(1)}$ is the linear susceptibility for the probe field. We take the probe field to be polarized in the $z$-direction, $\mathbf{E}_{1}=E_{1}(t, x, y) \hat{\mathbf{z}}$. Its linearized wave equation may now 
be written as

$$
\frac{\partial^{2} E_{1}}{\partial x^{2}}+\frac{\partial^{2} E_{1}}{\partial y^{2}}-\frac{1}{v_{p}^{2}}\left(1+2 \epsilon_{1}\right) \frac{\partial^{2} E_{1}}{\partial t^{2}}=0 .
$$

Here

$$
v_{p}=\frac{1}{\sqrt{1+\chi_{p}^{(1)}}}=\frac{1}{n_{p}},
$$

is the speed of a probe pulse in the medium with index of refraction $n_{p}$ due to linear effects, and

$$
\epsilon_{1}=\gamma_{j} E_{0}^{j}
$$

with

$$
\gamma_{j}=\frac{1}{n_{p}^{2}}\left(\frac{\chi_{z z j}^{(2)}+\chi_{z j z}^{(2)}}{2}\right)
$$

Equation (13) describes the propagation of a probe pulse, $E_{1}(t, x, y)$, on a space and time dependent background determined by $E_{0}$. The phase velocity of the probe field will now be given by, assuming $\left|\epsilon_{1}\right| \ll 1$,

$$
v_{p h}=\frac{v_{p}}{\sqrt{1+2 \epsilon_{1}}} \approx v_{p}\left(1-\epsilon_{1}\right)
$$

which depends upon the value of the background field, $\mathbf{E}_{0}$. The justification for the assumption that $\left|\epsilon_{1}\right|$ is small will be found later when we derive the magnitude of the fractional flight time variations. If we form wavepackets over a frequency range where dispersion is small, then $v_{p h}$ is also the group velocity of the packets. For a slab of material with thickness $d$, the flight time of a pulse propagating in the $x$-direction will be $d / v$ without the background field, and approximately

$$
t_{d}=n_{p} \int_{0}^{d}\left(1+\epsilon_{1}\right) d x
$$

with the background field.

Note that although the total field $\mathbf{E}=\mathbf{E}_{0}+\mathbf{E}_{1}$ is a solution of a nonlinear equation, if the nonlinearity is weak, both $E_{0}$ and $E_{1}$ satisfy approximately linear equations to leading order. The primary effect of the nonlinearity is a coupling term, the term proportional to $\epsilon_{1}$ in Eq. (13). 


\section{VACUUM ELECTRIC FIELD FLUCTUATIONS}

So far our treatment has been in the classical level. We are interested in the fluctuations of the flight time of the pulse propagating in the slab of material. Fluctuations of the background field cause fluctuations in the speed of the probe field as well in $t_{d}$. The background field $E_{0}$ can be replaced by a quantum operator $\hat{E}_{0}$ and from Eq. (18) $t_{d}$ is replaced by $\hat{t}_{d}$. Here we take the fluctuations of $\hat{t}_{d}$ to be driven by the vacuum fluctuations of the quantized electric field. We will work in an approximation in which the various contributing modes of the quantized field propagate at approximately the same speed, and hence experience a frequency independent and isotropic index of refraction $n_{b}$, different from $n_{p}$.

\section{A. Correlation Functions}

We will need the electric field correlation functions in such a material, which may be obtained from the corresponding correlation functions in empty space by a simple argument. First consider the standing modes in a cavity of fixed geometry, and assume periodic boundary conditions. The spatial part of a mode function is independent of $n_{b}$, and will be proportional to $\mathrm{e}^{i \mathbf{k} \cdot \mathbf{x}}$. The temporal part will oscillate at an angular frequency $\omega=k / n_{b}$. The Faraday law, $\nabla \times \mathbf{E}=-\dot{\mathbf{B}}$, tells us that the magnitudes of the electric and magnetic fields of a mode are related by $B=(k / \omega) E=n_{b} E$. We require that the zero point energy of a given mode be $\omega / 2$, which implies

$$
\frac{1}{2} \omega=\frac{k}{2 n_{b}}=\frac{1}{2} \int d^{3} x\left(n_{b}^{2} E^{2}+B^{2}\right) .
$$

As a result, we have

$$
E \propto \frac{1}{n_{b}^{3 / 2}}, \quad B \propto \frac{1}{n_{b}^{1 / 2}},
$$

which agrees with Eqs. (1.31a) and (1.32) of Ref. [23]. This shows that the net effect on an electric field correlation function is an overall factor of $1 / n_{b}^{3}$ and a modification of the time dependence by $t \rightarrow t / n_{b}$, with no effect on the space dependence. This result may also be obtained from Eqs. (5) and (6) in Ref. [24]. Take the spatial separation to be in the 
$x$-direction, in which case the correlation functions become

$$
\left\langle E^{x}(x, t) E^{x}\left(x^{\prime}, t^{\prime}\right)\right\rangle=\frac{1}{\pi^{2} n_{b}^{3}\left[(\Delta x)^{2}-(\Delta t)^{2} / n_{b}^{2}\right]^{2}},
$$

and

$$
\left\langle E^{y}(x, t) E^{y}\left(x^{\prime}, t^{\prime}\right)\right\rangle=\left\langle E^{z}(x, t) E^{z}\left(x^{\prime}, t^{\prime}\right)\right\rangle=\frac{(\Delta x)^{2}+(\Delta t)^{2} / n_{b}^{2}}{\pi^{2} n_{b}^{3}\left[(\Delta t)^{2} / n_{b}^{2}-(\Delta x)^{2}\right]^{3}} .
$$

Here $\Delta x=x-x^{\prime}$ and $\Delta t=t-t^{\prime}-i \varepsilon$, and $\varepsilon>0$ makes the mode sums absolutely convergent and defines the location of the lightcone singularity. Note that the effective lightcone is given by the line $t=n_{b} x$, with $n_{b}>1$.

Note that for more general spatial separations, other components of the electric field correlation function can be nonzero, but are proportional to the spatial separations in the

$y$ and $z$ directions. For example, $\left\langle E^{x}(\mathbf{x}, t) E^{y}\left(\mathbf{x}^{\prime}, t^{\prime}\right)\right\rangle \propto \Delta x \Delta y$. However, these components are small if $\Delta y$ and $\Delta z$ are small.

\section{B. Dominance of Vacuum Fluctuations}

Here we wish to discuss our assumption that the root mean square of the background field, due to vacuum fluctuations, is large compared to that for the probe field, $\left|E_{0}\right| \gg\left|E_{1}\right|$, which was used in deriving Eq. (13). We consider two different ways to understand why this can be possible.

\section{Plane Wave Basis}

Here we consider the electric field operator to be expanded in a plane wave basis, with a narrow bandwidth of modes with frequencies near $\omega=\omega_{p}$ excited in a coherent state, and the remainder of the modes in their ground state. The former modes will constitute the probe field. The probe field modes are of higher frequency than the dominant vacuum modes, so this condition can still be satisfied for highly collimated probe beams with narrow bandwidths. For the sake of an estimate, we ignore the indices of refraction, and write $E_{1}^{2} \approx|z|^{2} \lambda_{p}^{-4}(\Delta \omega / \omega) \Delta \Omega$. Here $|z|^{2}$ is the mean number of photons per mode, $\Delta \omega / \omega$ is the 
fractional bandwidth, and $\Delta \Omega$ is the solid angle subtended by the probe beam. This is to be compared with Eq. (3), $E_{0}^{2}=a / \tau^{4}$. Even though $\lambda_{p}<\tau$, we can still have $\left|E_{1}\right| \ll\left|E_{0}\right|$ if $\Delta \omega / \omega \ll 1$ or $\Delta \Omega \ll 1$. This arises because fewer modes contribute to $\left|E_{1}\right|$ than to $\left|E_{0}\right|$.

\section{Wavepacket Basis}

An alternative, but equivalent approach involves an expansion of the electric field operator in a wavepacket basis. Let $\left\{\mathbf{f}_{j}\right\}$ be a complete set of wavepacket modes for the electric field. We can expand the electric field operator as

$$
\mathbf{E}(\mathbf{x}, t)=\sum_{j}\left(a_{j} \mathbf{f}_{j}+a_{j}^{\dagger} \mathbf{f}_{j}^{\star}\right)
$$

Here $j=(\lambda, \ell)$, where $\lambda$ labels the polarization, and $\ell$ labels the wavepacket mode function, which depends upon space and time. The normalization of the $\mathbf{f}_{j}$ is the same as that for a mode of $\dot{\phi}=\partial \phi / \partial t$, where $\phi$ is a massless scalar field with

$$
\phi=\sum_{\ell}\left(a_{\ell} h_{\ell}+a_{\ell}^{\dagger} h_{\ell}^{\star}\right)
$$

and $h_{\ell}(\mathbf{x}, t)$ are wavepacket modes with unit Klein-Gordon norm:

$$
\left\|h_{\ell}\right\|=1=i \int d^{3} x\left(h_{\ell}^{\star} \dot{h}_{\ell}-\dot{h}_{\ell}^{\star} h_{\ell}\right)
$$

Thus we can set $\mathbf{f}_{j}=\mathbf{f}_{(\lambda, \ell)}=\hat{\mathbf{e}}_{\lambda} \dot{h}_{\ell}$, where $\hat{\mathbf{e}}_{\lambda}$ is a unit polarization vector.

Consider the quantized electric field to be in a state where all modes except $j=p$, the probe field wavepacket, are in the ground state, and $j=p$ is in a coherent state of amplitude z. Write $\mathbf{E}=\mathbf{E}_{0}+\mathbf{E}_{1}$, where $\mathbf{E}_{1}=a_{p} \mathbf{f}_{p}+a_{p}^{\dagger} \mathbf{f}_{p}^{\star}$. Then $a_{p}|z\rangle=z|z\rangle$ leads to

$$
\left\langle z\left|\mathbf{E}_{1}^{2}\right| z\right\rangle=\left(z \mathbf{f}_{p}+z^{\star} \mathbf{f}_{p}^{\star}\right)^{2}+\left|\mathbf{f}_{p}\right|^{2}=4\left[\operatorname{Re}\left(z \dot{h}_{p}\right)\right]^{2}+\left|\dot{h}_{p}\right|^{2}
$$

Next we need to estimate the magnitude of the squared mode function in this expression. If $h_{p}$ is a wavepacket sharply peaked in a frequency near $\omega=\omega_{p}$ then $\dot{h}_{p} \approx-i \omega_{p} h_{p}$ and we may use Eq. (25) to write

$$
\left\|h_{p}\right\|=1 \approx 2 \omega_{p} v_{p}\left(\left|h_{p}\right|_{\mathrm{rms}}\right)^{2}
$$


where $v_{p}$ is the approximate spatial volume of the wavepacket and $\left|h_{p}\right|_{\mathrm{rms}}$ is the root mean squared value of $\left|h_{p}\right|^{2}$ averaged in space. We wish to use the spatial average of $\left\langle z\left|\mathbf{E}_{1}^{2}\right| z\right\rangle$ as a measure of $E_{1}^{2}$. Assume $|z|>1$, so on average there are several photons in the wavepacket mode and we can ignore the zero point contribution in Eq. (26). We estimate the spatial average of $4\left[\operatorname{Re}\left(z \dot{h}_{p}\right)\right]^{2}$ to be of order $|z|^{2} \omega_{p}^{2}\left(\left|h_{p}\right|_{\mathrm{rms}}\right)^{2}$ and write

$$
E_{1}^{2} \approx|z|^{2} \omega_{p}^{2}\left(\left|h_{p}\right|_{\mathrm{rms}}\right)^{2} \approx \frac{|z|^{2} \omega_{p}}{2 v_{p}}
$$

Now consider the time average of $E_{0}^{2}$ which is approximately given by Eq. (3), and recall that we are ignoring indices of refraction for the purpose of an estimate. The root mean squared value of $E_{1}$ is small compared to that for $E_{0}$ if $\left\langle 0\left|\bar{E}_{0}^{2}\right| 0\right\rangle \gg E_{1}^{2}$, or if

$$
\frac{a}{\tau^{4}} \gg \frac{|z|^{2} \omega_{p}}{v_{p}} .
$$

Let the volume $v_{p}=N \lambda_{p}^{3}$ where $N \gg 1$. Note that $N$ is expected to be large for two reasons: First, the length of the wavepacket in the propagation direction must be several times $\lambda_{p}$, and the size of the wavepacket in the transverse direction also must be several times $\lambda_{p}$ and it can be much larger. Recall that $\tau \approx d$ the thickness of the slab, so we need:

$$
d \lesssim\left(\frac{a N}{2 \pi|z|^{2}}\right)^{1 / 4} \lambda_{p}
$$

This can be satisfied by $\lambda_{p} \ll d$ if $N$ is large. Recall that the zero point contribution of the

probe wavepacket to $E_{1}$ can be ignored if there are several photons in this mode. Similarly, its contribution to $E_{0}$ can also be ignored if Eq. (30) is satisfied.

Here it is important to note that we take the condition $\left|E_{0}\right| \gg\left|E_{1}\right|$ to apply to the root mean squares, not to mean values. In fact, here $\left\langle 0\left|\mathbf{E}_{0}\right| 0\right\rangle=0$ and $\left\langle z\left|\mathbf{E}_{1}\right| z\right\rangle \neq 0$. The background field is undergoing large amplitude fluctuations around a mean value of zero, but its mean squared value is large compared to that for the probe field.

\section{FLIGHT TIME VARIANCE}

We are considering the case where the probe pulse is in a higher frequency band than the dominant vacuum modes, and has an index of refraction of $n_{p}>n_{b}$. Thus the probe pulse 


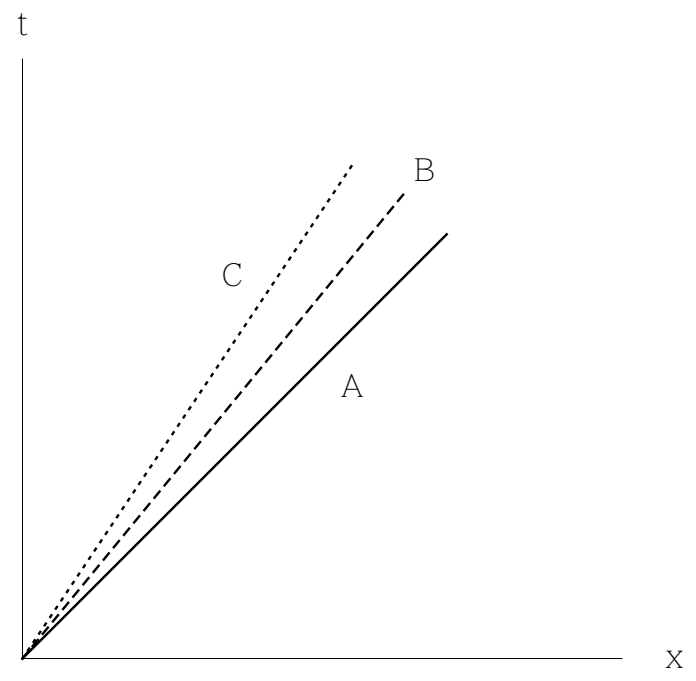

FIG. 1: Here $A$ is the true lightcone $(t=x), B$ is the effective lightcone $\left(t=n_{b} x\right)$, and $C$ is the worldline of the probe pulse $\left(t=n_{p} x\right)$.

travels on a worldline which lies inside the effective lightcone, which is turn inside the true lightcone, as illustrated in Fig. 1. The travel time of a pulse through a slab of material is given by an integral of the form of that in Eq. (18). However, this travel time undergoes fluctuations around a mean value of $\left\langle t_{d}\right\rangle=d / v$, and with a variance of

$$
(\delta t)^{2}=\left\langle t_{d}^{2}\right\rangle-\left\langle t_{d}\right\rangle^{2}=n_{p}^{2} \int_{0}^{d} d x \int_{0}^{d} d x^{\prime}\left\langle\epsilon_{1}(x, t) \epsilon_{1}\left(x^{\prime}, t^{\prime}\right)\right\rangle .
$$

The correlation function $\left\langle\epsilon_{1}(x, t) \epsilon_{1}\left(x^{\prime}, t^{\prime}\right)\right\rangle$ may be expressed in terms of the electric field correlation functions given in Eqs. (21) and (22). The integration in Eq. (31) is along the path of the probe pulse, defined by $x=t / n_{p}$, and illustrated in Fig. 1.

The integrals of the electric field correlation functions will be well defined provided there is a sampling function which falls smoothly to zero at both ends of the integration range. In the present context, such a function can be provided by the geometry of the slab of nonlinear material. Suppose that the density of this material is tapered gradually at both ends, so that $\chi_{i j k}^{(2)}$ can be replaced by $\chi_{i j k}^{(2)} g(x)$, where $g(x)$ is a profile function of width $d$, and $\int_{-\infty}^{\infty} d x g(x)=d$, illustrated in Fig. 2. This profile function, along with the worldline of the probe pulse, define a temporal sampling function, $f_{\tau}(t)=g\left(t / n_{p}\right) / \tau$, whose characteristic width is $\tau=n_{p} d$. We take the normalization of $f_{\tau}(t)$ to be defined by Eq. (2). The effect 


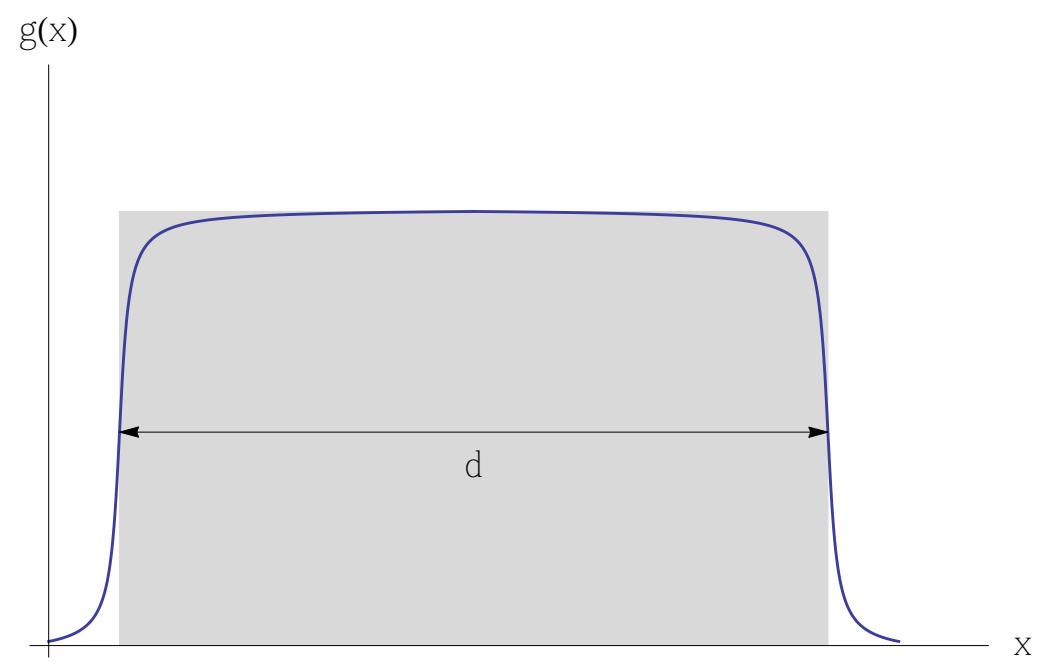

FIG. 2: The profile function $g(x)$ is illustrated.

of the profile function is to insert a factor of $g(x) g\left(x^{\prime}\right)$ in the integrand of Eq. (31), and to extend the range of integration to all $x$.

The fractional variance in flight time may be expressed as

$$
\delta^{2}=\frac{(\delta t)^{2}}{\left\langle t_{d}\right\rangle^{2}}=\int_{-\infty}^{\infty} d t \int_{-\infty}^{\infty} d t^{\prime} f_{\tau}(t) f_{\tau}\left(t^{\prime}\right) \gamma_{j} \gamma_{k}\left\langle E^{j}(x, t) E^{k}\left(x^{\prime}, t^{\prime}\right)\right\rangle
$$

where $x=t / n_{p}$ and $x^{\prime}=t^{\prime} / n_{p}$. If we use Eqs. (21) and (22), we find

$$
\delta^{2}=\frac{C n_{b}}{\pi^{2}\left(n_{p}^{2}-n_{b}^{2}\right)^{3} d^{4}}\left[\left(n_{p}^{2}-n_{b}^{2}\right) \gamma_{x}^{2}+\left(n_{p}^{2}+n_{b}^{2}\right)\left(\gamma_{y}^{2}+\gamma_{z}^{2}\right)\right] .
$$

Here we have used $d=\tau / n_{p}$ and the result

$$
\int_{-\infty}^{\infty} d t \int_{-\infty}^{\infty} d t^{\prime} \frac{f_{\tau}(t) f_{\tau}\left(t^{\prime}\right)}{\left(t-t^{\prime}-i \epsilon\right)^{4}}=\frac{C}{\tau^{4}}
$$

Here $C$ is a dimensionless constant whose value depends upon the choice of the sampling function $f_{\tau}(t)$. If this function is a Lorentzian, Eq. (4), then $C=1 / 16$. In deriving Eq. (33), we have also assumed that $\left\langle E^{j}(x, t) E^{k}\left(x^{\prime}, t^{\prime}\right)\right\rangle \approx 0$ for $j \neq k$. This should be justified if the size of the wavepackets in the transverse directions is small compared to $d$, the length of the flight path in the $x$ direction. This will make the contribution of the off-diagonal components of the electric field correlation function small compared to those given in Eqs. (21) and (22). (Recall the discussion at the end of Sec. III A.) 
The root mean square of the fractional flight time variation is

$$
\delta_{\mathrm{rms}}=\sqrt{\delta^{2}} \propto \frac{\chi^{(2)}}{d^{2}},
$$

where $\chi^{(2)}$ is a component of the $\chi_{i j k}^{(2)}$ tensor, which has typical values of the order of $10^{-12} \mathrm{~m} / \mathrm{V}$ in SI units. The dimensionless ratio, $\chi^{(2)} / d^{2}$, may be expressed as

$$
\frac{\chi^{(2)}}{d^{2}}=6.0 \times 10^{-8}\left[\frac{\chi^{(2)}}{10^{-12} m / V}\right]\left(\frac{1 \mu m}{d}\right)^{2} .
$$

We may see from Eq. (5) that $\chi^{(2)}$, and hence the $\gamma_{i}$, have dimensions of length squared in our units. In writing Eq. (36), we have used $1 V \approx 1.67 \times 10^{7} \mathrm{~m}^{-1}$. This conversion factor may be derived from the relations $\hbar=c=1$ and $\epsilon_{0} \approx 8.85 \times 10^{-12} \mathrm{C}^{2} /\left(\mathrm{N} \mathrm{m}^{2}\right)=1$ in our units.

A specific example of a material is that of Cadmium selenide (CdSe), which has $\chi_{z z z}^{(2)} \approx$ $1.1 \times 10^{-10} \mathrm{~m} / \mathrm{V}$ at a wavelength of $10.6 \mu \mathrm{m}$ [25], and indices of refraction of $n_{p}=2.54$ at $\lambda=\lambda_{p}=1.06 \mu m$ and of $n_{b}=2.43$ (ordinary ray) and $n_{b}=2.44$ (extraordinary ray) at $\lambda=\lambda_{b}=10.6 \mu m[26,27]$. The nearly equal values of $n_{b}$ for the ordinary and extraordinary rays justifies the isotropy assumption in Eq. (8). The other components of $\chi_{i j k}^{(2)}$, which appear in Eq. (33), by means of $\gamma_{x}$ and $\gamma_{y}$, vanish. The crystal lattice of this material has hexagonal symmetry, and hence a single axis of rotational symmetry, which is taken to be the $z$-axis. With $C=1 / 16$, we find the estimate

$$
\delta_{\text {rms }}=1.1 \times 10^{-8}\left(\frac{10 \mu m}{d}\right)^{2} .
$$

This is an estimate of the fractional variation in flight times of wavepackets peaked at a mean wavelength of $\lambda_{p} \approx 1 \mu \mathrm{m}$ traversing a distance $d$. Clearly the spatial spread in the wavepackets is relevant here. The bandwidth in angular frequency cannot be more that about $\Delta \omega \approx 2 \pi / \lambda_{p}$ with a corresponding spatial spread of $\Delta x=1 / \Delta \omega=\lambda_{p} / 2 \pi$. With $d=10 \mu m$, a single pulse could measure a flight time to an accuracy of about $2 \times 10^{-2}$ at best. However, averaging over a very large number of pulses might lead to an accurate determination of $\delta_{\mathrm{rms}}$, which is an uncertainty in flight time due to vacuum fluctuations. If it is possible to do experiments with smaller slabs, and correspondingly shorter wavelengths, 
then the effect could be larger, due to the $1 / d^{2}$ dependence of $\delta_{\text {rms }}$. More detailed studies of the observability of switched vacuum fluctuations is a topic for future work. Note that the small value for our estimate of $\delta_{\text {rms }}$ justifies the assumption made in Eq. (17) that $\left|\epsilon_{1}\right| \ll 1$.

\section{SUMMARY AND DISCUSSION}

In this paper we have presented a model in which vacuum electromagnetic field fluctuations can potentially produce variations in the flight times of probe pulses through a material with a nonzero second order susceptibility. The key idea is that the nonlinearity can cause the probe pulse to satisfy Eq. (13), where the speed of the probe pulse depends upon the background field. From this equation, it follows that fluctuations of the background field lead to fluctuations in the effective speed of a probe pulse wavepacket. This effect is closely analogous to the lightcone fluctuation effects expected in quantum gravity. Here the background field consists of the switched vacuum fluctuations of the electromagnetic field. The probe field is a wavepacket mode in a coherent state which responds to a fluctuating index of refraction driven by the vacuum fluctuations. Although the mean electric field in the vacuum state is zero, its mean square, given by Eq. (3), can be large compared to the mean square of the classical probe field.

The model presented here may be extended to include the effects of squared electric field fluctuations. Here we give a brief account, and a more detailed version will be presented elsewhere [28]. If we include third-order polarizability terms in Eq. (5), then the wave equation for the probe field, Eq. (13), will contain a term $\epsilon_{2} \propto \chi^{(3)}\left(E_{0}\right)^{2}, \quad[14,15]$, where $\chi^{(3)}$ is a component of the third-order polarizability tensor, $\chi_{i j k l}^{(3)}$, and $E_{0}$ is a component of the background electric field. Now the probe pulse is sampling the squared electric field operator on a timescale $\tau$. Let $E^{2}$ denote the normal order of the squared electric field operator, and $\bar{E}^{2}$ denote its time average, defined as in Eq. (1). Now the analog of Eq. (3) is

$$
\left\langle 0\left|\left(\bar{E}^{2}\right)^{2}\right| 0\right\rangle=\frac{b}{\tau^{8}},
$$


where $b$ is a dimensionless constant determined by the sampling function. We can make an approximate estimate of the magnitude of the squared electric field fluctuations by simply replacing $\chi^{(2)} \rightarrow \chi^{(3)}$ and $d \rightarrow d^{2}$ in our previous result, Eq. (35). Typical values of $\chi^{(3)}$ are of order $10^{-18} \mathrm{~m}^{2} / V^{2}$ in SI units. Thus our estimate for the fractional variation in flight time becomes

$$
\delta_{\mathrm{rms}} \approx \frac{\chi^{(3)}}{d^{4}}=3.6 \times 10^{-13}\left(\frac{\chi^{(3)}}{10^{-18} m^{2} / V^{2}}\right)\left(\frac{10 \mu m}{d}\right)^{4} .
$$

Thus the effects of $E^{2}$ fluctuations are typically small compared to those of electric field fluctuations, but the former grow more rapidly with decreasing $d$ and hence decreasing sampling time. It is also of interest to note that the probability of large $\bar{E}^{2}$ fluctuations can be much larger than for electric field fluctuations. In contrast to the Gaussian probability distribution of $\bar{E}$, the corresponding distribution for $\bar{E}^{2}$ falls approximately as [29]

$$
P(x) \sim x^{-2} \mathrm{e}^{-x^{1 / 3}}, \quad x \gg 1
$$

where $x=\bar{E}^{2}\left(4 \pi \tau^{2}\right)^{2}$ is a dimensionless measure of $\bar{E}^{2}$, and Lorentzian averaging is assumed. This means that large fluctuations are less rare than for a Gaussian probability distribution. The distribution of flight times due to electric field fluctuations, with the variance described by Eq. (37), is Gaussian, while the corresponding distribution due to squared electric field fluctuations will be a non-Gaussian distribution of the type discussed in Ref. [29], with a long positive tail.

In this paper, we have argued that vacuum fluctuations of the quantized electric field can lead to fluctuations in the flight times of pulses in a nonlinear material with nonzero second order susceptibility, and have made some estimates of the magnitude of this effect. The model presented here is an analog model for quantum lightcone fluctuations, as was the model of Ref. [15]. However, in the latter model, the fluctuations were not vacuum fluctuations, but were generated by a squeezed state of the electromagnetic field. The model described here is an illustration of the reality of temporally switched vacuum fluctuations, where the details of the switching are given by the geometry of the slab of nonlinear material. The probe pulse is only sensitive to vacuum fluctuations occurring in a finite time interval, 
so a nonzero effect arises, despite the tendency of vacuum fluctuations to be anticorrelated. This model provides an explicit physical meaning to test functions for quantized fields, and demonstrates how time sampling of vacuum fluctuations can have potentially observable effects.

\section{Acknowledgments}

This work was supported in part by the National Science Foundation under Grant PHY1205764, and by the Brazilian research agencies CNPq (245985/2012-3 and 304486/2012-4), FAPEMIG, and CAPES under scholarship BEX 18011/12-8.

[1] C. M. Wilson, G. Johansson, A. Pourkabirian, J. R. Johansson, T. Duty, F. Nori, and P. Delsing, Nature 479 (2011) 376-379, arXiv:1105.4714.

[2] L. Parker, Phys. Rev. 183 (1969) 1057-1068.

[3] S. A. Fulling and P. C. W. Davies, Proc. R. Soc. London A348 (1976) 393-414.

[4] P. C. W. Davies and S. A. Fulling, Proc. R. Soc. London A356 (1977) 237-257.

[5] L. H. Ford and A. Vilenkin, Phys. Rev. D 25 (1982) 2569-2575.

[6] K. K. Boddy, S. M. Carroll, and J. Pollack, arXiv:1405.0298.

[7] R. L. Jaffe, Phys. Rev. D 72 (2005) 021301, hep-th/0502158.

[8] H. Yu and L. H. Ford, Phys. Rev. D 70 (2004) 065009, quant-ph/0406122.

[9] C. H. G. Bessa, V. B. Bezerra, and L. H. Ford, J. Math. Phys. 50 (2009) 062501, arXiv:0804.1360.

[10] V. Parkinson and L. H. Ford, Phys. Rev. A 84 (2011) 062102, arXiv:1106.6334.

[11] V. Parkinson and L. H. Ford, Phys. Rev. D 89 (2014) 064018, arXiv:1311.6422.

[12] R. F. Streater and A. S. Wightman, PCT, Spin and Statistics, and All That, Benjamin, New York, 1964, p 97.

[13] H. Huang and L. H. Ford, Phys. Rev. D, in press, arXiv:1503.02962.

[14] C. H. G. Bessa, V. A. De Lorenci, and L. H. Ford, Phys. Rev. D 90 (2014) 024036, arXiv:1402.6285. 
[15] L. H. Ford, V. A. De Lorenci, G. Menezes, and N. F. Svaiter, Ann. Phys. 329 (2013) 80-92, arXiv:1202.3099.

[16] W. Pauli, Helv. Phys. Acta. Suppl. 4 (1956) 69. This reference consists of some remarks made by Pauli during the discussion of a talk by O. Klein at a 1955 conference in Bern, on the 50th anniversary of relativity theory.

[17] S. Deser, Rev. Mod Phys. 29 (1957) 417-422.

[18] B. S. DeWitt, Phys. Rev. Lett. 13 (1964) 114-117.

[19] L. H. Ford, Phys. Rev. D 51 (1995) 1692-1700, arXiv:gr-qc/9410047.

[20] L. H. Ford and N. F. Svaiter, Phys. Rev. D 54 (1996) 2640-2646, arXiv:gr-qc/9604052.

[21] L. H. Ford and N. F. Svaiter, Phys. Rev. D 56 (1997) 2226-2235, arXiv:gr-qc/9704050.

[22] R. W. Boyd, Nonlinear optics, 3rd ed., Academic Press, New York, 2008.

[23] R. J. Glauber and M. Lewenstein, Phys. Rev. A 43 (1991) 467-491.

[24] S. M. Barnett, B. Huttner, and R. Loudon, Phys. Rev. Lett. 68 (1992) 3698-3701.

[25] A. Penzkofer, M. Schäffner, and X. Bao, Opt. and Quantum Electron. 22 (1990) 351-367.

[26] R. L. Herbst and R. L. Byer, App. Phys. Lett. 19 (1971) 527-529.

[27] G. C. Bhar, App. Optics 15 (1976) 305-307.

[28] C. H. G. Bessa, V. A. De Lorenci, and L. H. Ford, manuscript in preparation.

[29] C. J. Fewster, L. H. Ford, and T. A. Roman, Phys. Rev. D 85 (2012) 125038, arXiv:1204.3570. 